\title{
Polarized thermal dust emission as seen by Planck: A comparison with MHD simulations and lessons from a toy model
}

\author{
F. Levrier ${ }^{1}$ on behalf of the Planck collaboration and J. Neveu ${ }^{1}$ \\ ${ }^{1}$ LERMA, Observatoire de Paris, PSL Research University, CNRS, Sorbonne Universités, \\ UPMC Univ. Paris 06, École Normale Supérieure, F-75005, Paris, France \\ email: francois.levrier@ens.fr
}

\begin{abstract}
The Planck satellite has mapped the polarized microwave sky (from $30 \mathrm{GHz}$ to 353 $\mathrm{GHz}$ ) with unprecedented sensitivity and angular resolution. This wealth of data yields the first complete map of polarized thermal emission from dust in our own Galaxy, shedding new light on the formation of dense cold structures within which new stars and planetary systems are born, under the combined effects of gravity, turbulence and magnetic fields. We present a statistical analysis of this polarized emission from nearby molecular clouds, with an emphasis on the evolution of the maximum polarization fraction observed as a function of column density, and on the anti-correlation between the polarization fraction and the local dispersion of polarization angles. To interpret this data, numerical simulations of anisotropic MHD turbulence underline the essential role played by the topology of the interstellar magnetic field, in particular its largescale component. As an extension to this work published in Planck Intermediate Results XX (A\&A, 576, 105, 2015), the statistical properties of the random component of the interstellar magnetic field are explored using a toy model based on fractional Brownian motion (fBm) fields.
\end{abstract}

Keywords. ISM: clouds, ISM: magnetic fields, submillimeter

\section{Introduction}

The Planck satellite (Planck 2015 Results I) has mapped the entire microwave sky with unprecedented sensitivity from $30 \mathrm{GHz}$ to $857 \mathrm{GHz}$. Its polarimetric capabilities up to 353 $\mathrm{GHz}$ have also given us the first complete map of polarized thermal dust emission from our Galaxy. This map yields precious information on the geometry of the Galactic magnetic field, its interaction with structures of matter, and gives strong constraints for models of interstellar dust.

\section{Statistical properties of polarized dust emission maps from nearby molecular clouds}

In Planck Intermediate Results XX (2015), we have analyzed the Planck polarized thermal dust emission maps at $353 \mathrm{GHz}$ towards a set of ten nearby molecular clouds, focusing on the observed anti-correlations (1) between the total gas column density $N_{\mathrm{H}}$ and the maximum polarization fraction $p_{\max }$ at a given $N_{\mathrm{H}}$, and (2) between the polarization fraction $p$ and the local dispersion of polarization angles, which is quantified by the polarization angle dispersion function $\mathcal{S}$ (Planck Intermediate Results XIX, 2015). For the less diffuse fields (Polaris Flare, Taurus, Orion, Ophiuchus, Chamaeleon-Musca), we find that the first anti-correlation is $p_{\max }=$ $m \log \left(N_{\mathrm{H}} / \mathrm{cm}^{-2}\right)+c$ with $m \sim-0.1$ and $c \sim 2-3$, and that the second one is $\log \left(S /{ }^{\circ}\right)=$ $m^{\prime} \log p+c^{\prime}$ with $m^{\prime} \sim-0.9$ and $c^{\prime} \sim-0.3$. 


\section{Comparison with MHD simulations}

To examine the role of the magnetic field in these anti-correlations (as opposed to the possible role played by dust alignement changes in various environments), we extract a $18 \mathrm{pc} \times 18 \mathrm{pc} \times 18$ pc subset from a RAMSES (Teyssier, 2002, Fromang et al.2006, Hennebelle et al.2008) simulation of magnetized converging flows of warm $(8000 \mathrm{~K})$ atomic gas, which condense into dense cold structures near the mid-plane. A magnetic field, initially along the gas flows, permeates the medium. We rotate the MHD simulation cube, place it $100 \mathrm{pc}$ away and simulate Stokes $I$, $Q, U$ maps by integrating along the line of sight. Resulting maps are smoothed at 15 FWHM and simulated polarization fractions $p$ and angles $\psi$ are derived, as well as the angle dispersion function $\mathcal{S}$.

We find that these simulations reproduce very well the decrease of maximum polarization fractions with increasing column densities, with very similar coefficients. This shows that this trend is primarily linked to the turbulent structure of the magnetic field, i.e. an accumulation of variously polarized structures along the line of sight. Polarization fractions are also found to anti-correlate with $\mathcal{S}$, but the latter is larger in the simulations than in the observations at a given $p$. This points to a possible shortcoming of these numerical simulations.

\section{A toy model for accessing the statistical properties of the magnetic field}

In order to gain insight on the statistical properties of the random component of the interstellar magnetic field, we build synthetic observations of polarized dust emission using dust density and magnetic field cubes with controlled one- and two-point statistics. Exploring the range of physical parameters (spectral indices of the dust density field and of the magnetic field components, level of density fluctuations, ratio of turbulent to regular magnetic field, and physical depth of the model cloud), we build a database relating these to many observables derived from the simulated Stokes maps. We can then infer physical parameters from actual observations based on a least-squares analysis.

\section{Conclusions}

Towards nearby molecular clouds, the polarization of dust thermal emission at the scales observed by Planck is essentially related to the geometry of the magnetic field. Observed anticorrelations are well reproduced by MHD simulations of the diffuse ISM, with comparable correlation coefficients. To go beyond this first analysis, we pursue a least-squares analysis to retrieve the statistical properties of the interstellar magnetic field from Planck observations. Application of this method on a toy model of the turbulent magnetized ISM shows good promise, and we are currently working towards its application on Planck data.

\section{References}

Fromang, S., Hennebelle, P., Teyssier, R. 2006, A\&SA, 457, 371

Hennebelle, P., Banerjee,R., Vazquez-Semadeni, E., Klessen, R.S., Audit, E. 2008, A\&্A, 486, L43

Planck 2015 Results I 2015, A\& $A$, submitted

Planck Intermediate Results XIX 2015, $A \& A, 576,104$

Planck Intermediate Results XX 2015, $A \mathscr{E} A$ A, 576, 105

Teyssier, R. 2002, A\&GA, 385, 337 